\title{
Produtividade, adaptabilidade e estabilidade fenotípica de cultivares de tomateiro sob diferentes condições de ambiente ${ }^{(1)}$
}

\begin{abstract}
Ronan Gualberto ${ }^{(2)}$, Leila Trevizan $\mathrm{Braz}^{(3)}$ e David Ariovaldo Banzatto(4)
Resumo - O objetivo deste trabalho foi estudar o desempenho produtivo, adaptabilidade e estabilidade fenotípica de seis genótipos de tomateiro na região de Marília, SP. Os experimentos foram conduzidos em nove ambientes (seis sob condições de cultivo protegido e três sob condições de céu aberto), com seis genótipos (Carmen, Diva, Donador, Graziela, Vita e HE-295), em blocos casualizados, com quatro repetições. Ocorreram diferenças significativas entre ambientes, e a média geral dos cultivos protegidos superou a dos cultivos a céu aberto quanto à produtividade, apesar de a média geral dos cultivos a céu aberto ser superior quanto ao peso médio de frutos. As cultivares, à exceção de HE-295, demonstraram alta estabilidade, merecendo destaque as cultivares Carmen, Donador e Vita, que tiveram rendimento médio superior ao da média geral, adaptabilidade geral e comportamento previsível em todos os ambientes estudados. Quanto ao peso médio dos frutos, as cultivares Diva e Vita foram as únicas que mostraram ampla adaptabilidade a todos os ambientes, comportamento previsível, além de apresentarem peso médio do fruto superior ao da média geral.
\end{abstract}

Termos para indexação: Lycopersicon esculentum, interação genótipo-ambiente, métodos de análise.

Productivity, adaptability and phenotypic stability of tomato cultivars grown under different environmental conditions

\begin{abstract}
The objective of this work was to evaluate productivity, adaptability and phenotypic stability of six tomato genotypes in Marília, SP, Brazil. The experiments were carried out at nine environments (six of them under conditions of protected cultivation and three under open air conditions), with six genotypes (Carmen, Diva, Donador, Graziela, Vita and HE-295), in a randomized block design, with four replications. There were significant differences among environments, independent of cultivation system. General average of protected cultivations overcame free cultivations considering yield, although the general average of free cultivations was overcame for mean fruit weights. All genotypes, except 'HE-295', showed high stability, with prominence for cultivars Carmen, Donador and Vita, which showed higher mean yield, and general adaptability and stability in all environments studied. For mean fruit weights, cultivars Diva and Vita were the only ones that showed wide adaptability for both environments and highly predictable behavior, with mean fruit weights superior to general average.
\end{abstract}

Index terms: Lycopersicon esculentum, genotype environment interaction, analytical methods.

(1) Aceito para publicação em 14 de dezembro de 2000.

Extraído da Tese de Doutorado do primeiro autor apresentada à Universidade Estadual Paulista (Unesp), Faculdade de Ciências Agrárias e Veterinárias (FCAV), Jaboticabal, SP.

(2) Universidade de Marília, Faculdade de Ciências Agrárias, Caixa Postal 554, CEP 17525-902 Marília, SP. E-mail: ronangual@uol.com.br

(3) Unesp, FCAV, Dep. de Produção Vegetal, Rod. Prof. Paulo Donato Castellane, s/n, CEP 14884-900 Jaboticabal, SP. E-mail: leilatb@fcav.unesp.br

(4) Unesp, FCAV, Dep. Ciências Exatas.

E-mail: banzatto@asbyte.com.br

\section{Introdução}

O tomateiro (Lycopersicon esculentum Mill.) constitui a mais universal das hortaliças. A versatilidade de uso, seja na forma natural ou industrializada, é considerada como um dos fatores responsáveis pela explosão mundial de seu consumo, no corrente século. É a hortaliça mais consumida no Brasil, superando a batata nos últimos anos (FAO, 2000). 
A produção de tomate para o consumo in natura no Brasil sofreu grandes transformações tecnológicas nesta última década. Um dos principais ajustes se refere à segmentação no campo das cultivares. Merece destaque o crescimento da nova geração de híbridos do grupo Salada, que substituíram as tradicionais cultivares japonesas de frutos exageradamente grandes e moles.

Os tomates do tipo longa-vida também têm mostrado expansão em ritmo mais acelerado do que se previa, sobretudo nas zonas de produção da região sudeste e sul do País. Desde a sua introdução no mercado brasileiro, em 1988, o tomate longa-vida tem aumentado a sua participação no mercado para consumo in natura. Estima-se que atualmente as cultivares de tomate longa-vida representam cerca de $70 \%$ do mercado (Della Vecchia \& Koch, 2000).

Em vista do manejo diferenciado, a produção de tomate em ambiente protegido, seja no sistema convencional ou hidropônico, demanda cultivares bem adaptadas e que proporcionem o máximo de rendimento de produtos de elevado padrão de qualidade.

Para obter o máximo de benefícios de cada um desses diferentes sistemas de cultivo, o conhecimento da interação genótipos $\mathrm{x}$ ambientes é fundamental, uma vez que ela se faz presente todas as vezes em que se testam diversas cultivares em diferentes condições ambientais.

Desde a década de 60 a interação genótipos $\mathrm{x}$ ambientes tem sido estudada em várias culturas, incluindo o tomate (Williams \& Gilbert, 1960). Os efeitos da interação genótipos $\mathrm{x}$ ambientes na cultura do tomateiro foram estudadas por Gull et al. (1984), Conti et al. (1990), Ortiz (1991) e Voican et al. (1995).

A identificação de cultivares com alta estabilidade é a estratégia mais amplamente empregada para atenuar os efeitos da interação genótipos x ambientes.

Existem vários métodos desenvolvidos para a caracterização de genótipos quanto à adaptabilidade e estabilidade, como os de Finlay \& Wilkinson (1963), Eberhart \& Russell (1966), Lin \& Binns (1988), Cruz et al. (1989) e Annicchiarico (1992), que têm como fundamento a interação genótipos $\mathrm{x}$ ambientes, que se distinguem nos conceitos de estabilidade adotados e em certos princípios estatísticos empregados.

São poucos os trabalhos que avaliaram a estabilidade de genótipos de tomate tanto para característi- cas quantitativas como qualitativas, podendo ser citados os de Poysa et al. (1986), Berry et al. (1988) e Ortiz \& Izquierdo (1994). No Brasil, foi encontrada apenas uma citação (Peixoto et al., 1999) sobre interação genótipos x ambientes para essa cultura.

O objetivo deste trabalho foi estudar o desempenho produtivo, adaptabilidade e estabilidade fenotípica de genótipos de tomateiro.

\section{Material e Métodos}

Foram utilizados dados de nove ensaios de competição entre cultivares de tomateiro, seis deles conduzidos sob condições de cultivo protegido (cinco no sistema convencional (SC) e um no sistema hidropônico (SH)) e três sob condições de céu aberto (CA), realizados na Fazenda Experimental Marcelo Mesquita Serva, da Universidade de Marília, Marília, SP, no período de 1997/98. As coordenadas do local são $22^{\circ} 12^{\prime} 50^{\prime \prime}$ de latitude Sul e $49^{\circ} 56^{\prime} 45^{\prime \prime}$ de longitude Oeste, e a altitude é de 610 metros.

Os ensaios constaram de cinco híbridos comerciais (Carmen, Diva, Donador, Graziela e Vita) e de um híbrido especial (HE-295) de tomateiro, do tipo Salada, sendo todos de hábito de crescimento indeterminado e comuns a todos os ambientes estudados (Tabela 1).

$\mathrm{O}$ delineamento experimental utilizado em todos os ensaios foi o de blocos ao acaso, com quatro repetições. Nas casas de vegetação, no sistema convencional, cada parcela foi constituída por linhas duplas com 12 plantas, no espaçamento de $0,50 \mathrm{~m}$ entre linhas, e $0,50 \mathrm{~m}$ entre plantas, sendo $1,0 \mathrm{~m}$ entre linhas duplas. No sistema hidropônico, cada parcela foi constituída por uma linha com 8 plantas, no espaçamento de $1,20 \mathrm{~m}$ entre linhas e $0,25 \mathrm{~m}$ entre plantas. No campo, cada parcela foi constituída por linhas duplas, com 14 plantas, no espaçamento

Tabela 1. Cultivares de tomateiro de mesa com as respectivas procedências e características. Unimar, Marília, 1997/ 1998.

\begin{tabular}{llll}
\hline Genótipos & Procedência & Fruto & Resistência $^{(1)}$ \\
\hline Carmen & Agroflora & Longa-vida & TMV, F, V e N \\
Diva & Rogers & Longa-vida & TMV, F, V, S e N \\
Donador & Bruinsma & Extra-firme & TMV, F, V e CL \\
Graziela R-190 & Zeraim Gedera & Longa-vida & TMV, F, V e CL \\
HE-295 & Agroceres & Extra-firme & TSWV, F, V e S \\
Vita & Petoseed & Longa-vida & TMV, F, V, S, SCL e N \\
\hline
\end{tabular}

(1)TSWV: vírus do vira-cabeça-do-tomateiro; TMV: vírus do mosaico-dotabaco; F: fusário; V: verticílio; S: estenfílio; CL: cladospório; SCL: esclerotínia; N: nematóide. 
de 1,00 m entre linhas, e 0,50 m entre plantas. Avaliaramse quatro plantas por parcela em todos os ensaios.

Os ciclos de cultivo de cada ambiente foram: $\mathrm{SC}_{1}$ : 27/5 a 10/11/97; $\mathrm{SC}_{2}$ : 5/8/97 a 5/1/98; $\mathrm{SC}_{3}$ : 4/2 a 10/8/98; $\mathrm{SC}_{4}: 6 / 5$ a $26 / 11 / 98 ; \mathrm{SC}_{5}: 25 / 6$ a $16 / 12 / 98 ; \mathrm{SH}: 2 / 2$ a 16/7/98; $\mathrm{CA}_{1}$ : $9 / 5$ a 10/11/97; $\mathrm{CA}_{2}: 4 / 2$ a 7/8/98; $\mathrm{CA}_{3}: 6 / 5$ a 12/11/98.

As mudas foram produzidas em bandejas de poliestireno expandido com 128 células piramidais, à exceção do ensaio conduzido no sistema hidropônico, em que as mudas foram produzidas no próprio sistema. Por ocasião do transplante, as adubações básicas, tanto nas casas de vegetação como no campo, foram feitas nas doses recomendadas (Makishima \& Miranda, 1995), de acordo com a análise química do solo (Tabela 2). No caso do sistema hidropônico, utilizou-se a solução nutritiva recomendada por Moraes (1997), cujos nutrientes (mg/L) são: $\mathrm{N}$ (nitratos) (168,6), N (amônio) (31,4), P (60), Ca (206), $\mathrm{Mg}(60), \mathrm{S}$ (sulfato) (150), B $(0,50), \mathrm{Cu}(0,10), \mathrm{Fe}(2,0)$, Mn $(0,75)$, Mo $(0,01)$ e Zn $(0,10)$. Na fase de berçário, a condutividade elétrica (CE) foi mantida na faixa de 1,5 a $2,0 \mathrm{mS} / \mathrm{cm}$, enquanto na fase de produção, na faixa de 2,5 a $3,5 \mathrm{mS} / \mathrm{cm}$, variando em função do desenvolvimento das plantas. $\mathrm{O}$ pH foi mantido na faixa de 5,5 a 6,5 nas duas fases, e tanto a $\mathrm{CE}$ quanto o $\mathrm{pH}$ foram monitorados diariamente.

Em todos os ensaios, as plantas foram conduzidas com uma haste, fazendo-se desbrotas laterais semanalmente e poda apical quando as plantas atingiam 2,0 $\mathrm{m}$ de altura.

As irrigações foram do tipo gotejamento nas casas de vegetação, e por sulcos, nos cultivos a campo. Os demais tratos culturais e fitossanitários foram efetuados na medida em que se fizeram necessários.

Foram avaliadas as características: produtividade de frutos comerciáveis (frutos com diâmetro transversal acima de $50 \mathrm{~mm}$ ) e peso médio de frutos comerciáveis.

Efetuou-se uma análise de variância em cada ensaio em separado, que teve como objetivo principal a determinação da variância residual de cada ensaio para posterior teste de homogeneidade. Posteriormente, realizou-se uma análise conjunta, envolvendo todos os ensaios, ou ambientes, sobre as características estudadas.

As estimativas da adaptabilidade e da estabilidade fenotípica foram obtidas segundo o método proposto por Eberhart \& Russell (1966), devido à sua aceitação universal entre pesquisadores que trabalham com diversas culturas, bem como à maior facilidade para se estimar e interpretar os parâmetros, considerando cada ensaio como um ambiente, independentemente do ano agrícola, ou do sistema de cultivo.

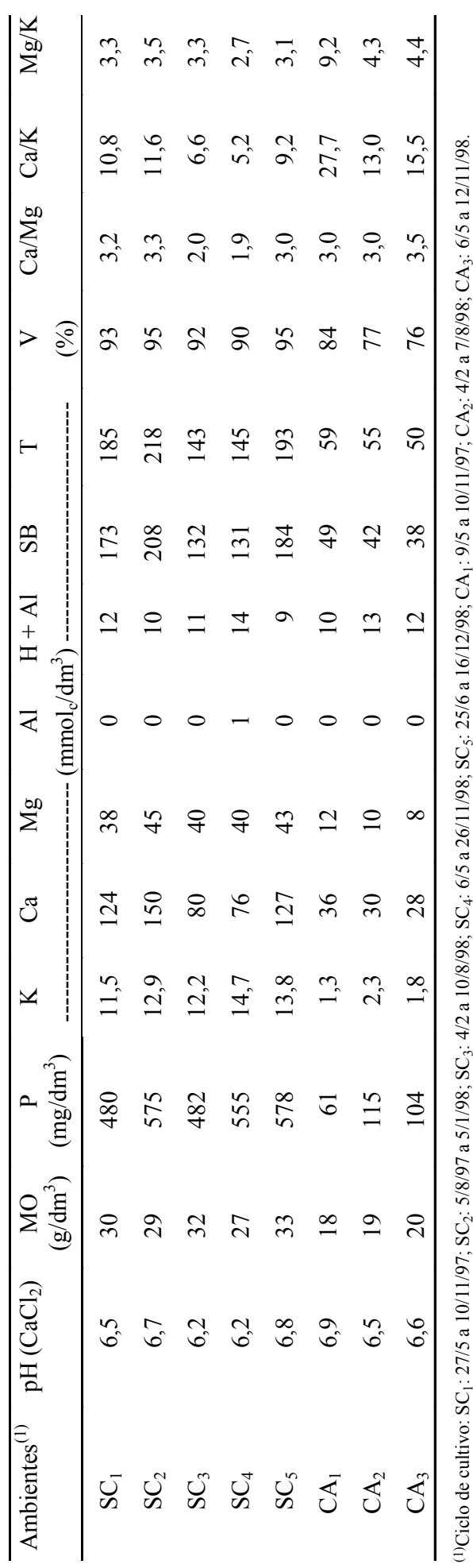

Pesq. agropec. bras., Brasília, v. 37, n. 1, p. 81-88, jan. 2002 
Neste método é adotado o seguinte modelo de regressão linear:

$Y_{i j}=\mu_{i}+b_{i} 1_{j}+\delta_{i j}+\varepsilon_{i j}$

em que: $Y_{\mathrm{ij}}$ é a média do genótipo i no ambiente $\mathrm{j} ; \mu_{\mathrm{i}}$ é a média do i-ésimo genótipo em todos ambientes; $b_{i}$ é o coeficiente de regressão linear, que mede a resposta do i-ésimo genótipo à variação do ambiente; $1_{j}$ é o índice ambiental, fornecido pela diferença entre a média do j-ésimo ambiente e a média geral de todas as cultivares em todos ambientes; $\delta_{\mathrm{ij}}$ é o desvio da regressão linear do i-ésimo genótipo no j-ésimo ambiente e $\varepsilon_{\mathrm{ij}}$ é o erro aleatório associado à observação $\mathrm{Y}_{\mathrm{ij}}$.

Em cada genótipo foi feita uma análise de regressão, utilizando-se o índice ambiental como variável independente e as características avaliadas como variável dependente. Assim, de acordo com o método proposto por Eberhart \& Russell (1966), o efeito do ambiente pode ser decomposto em dois componentes, um linear e outro nãolinear. O coeficiente de regressão $\left(b_{i}\right)$ está associado ao componente linear, indicando a adaptabilidade do genótipo, ou seja, sua capacidade de responder à melhoria do ambiente. Os desvios da regressão $\left(\mathrm{S}_{\mathrm{di}}^{2}\right)$ estão associados ao componente não-linear e indicam a estabilidade fenotípica. Um genótipo com $\mathrm{S}_{\mathrm{di}}^{2}=0$ tem comportamento previsível, de acordo com a grandeza do índice ambiental.

Assim, por este método, tem-se que um genótipo é estável quando $\mathrm{S}_{\mathrm{di}}^{2}=0$; não estável, quando $\mathrm{S}_{\mathrm{di}}^{2} \neq 0$; de adaptabilidade ampla, se $b_{i}=1$; adaptado a ambientes favoráveis, se $b_{i}>1$, e adaptado a ambientes desfavoráveis, se $b_{i}<1$. O coeficiente de determinação $\left(R^{2}\right)$ de cada genótipo foi usado como medida auxiliar na definição da estabilidade fenotípica e para quantificar que a proporção da variação em $Y_{\mathrm{ij}}$ é explicada pela regressão linear. A hipótese de que qualquer coeficiente de regressão não difere da unidade foi avaliada pelo teste t, e a hipótese de que os desvios da regressão de cada genótipo não diferem de zero foi verificada pelo teste F. Na realização das análises, foi utilizado o programa estatístico IGA (Departamento de Ciências Exatas, FCAV, Jaboticabal).

\section{Resultados e Discussão}

Ocorreram diferenças significativas entre as médias de produtividade nos diversos ambientes, independentemente do sistema de cultivo utilizado, indicando uma ampla faixa de variação nas condições ambientais onde foram conduzidos os ensaios (Tabela 3). Dentro de cada sistema de cultivo, diversos fatores como épocas de cultivo, temperatura, doenças, insetos-pragas e nematóides, foram respon-

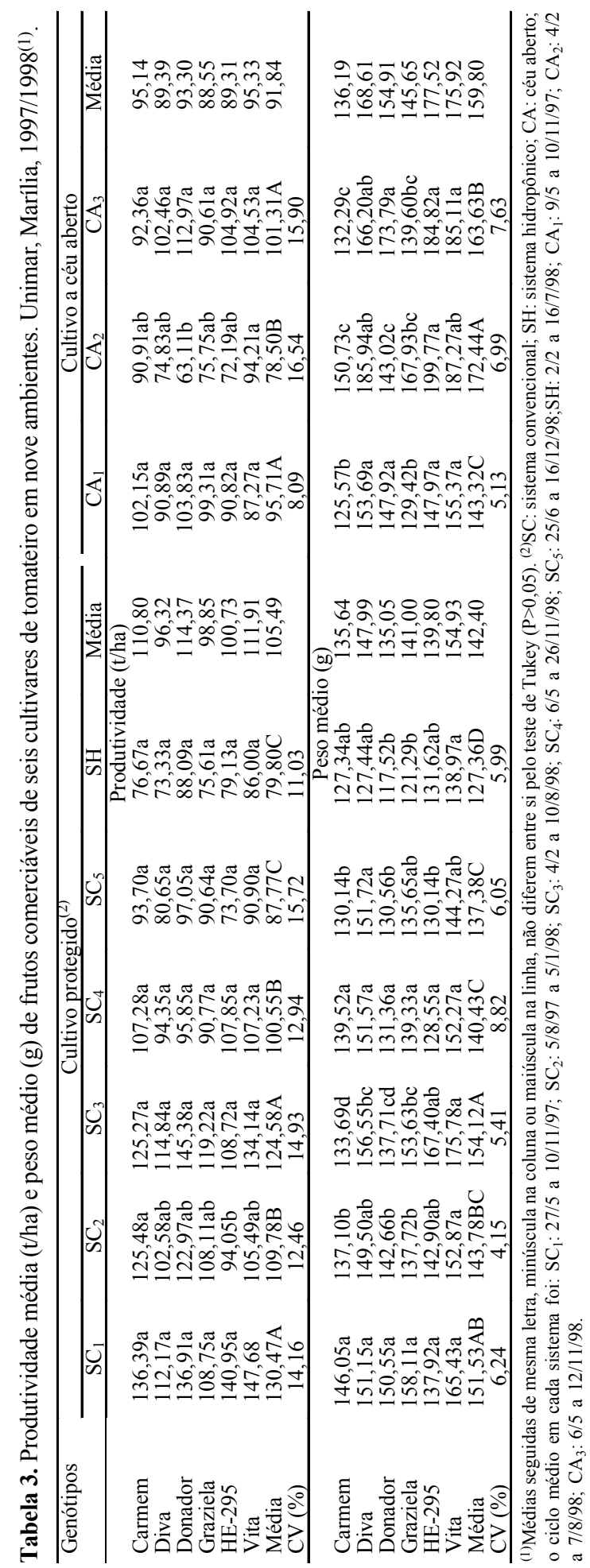


sáveis por essa ampla variação nas condições ambientais. No sistema de cultivo protegido, as diferenças entre o plantio no solo e sistema hidropônico também contribuíram fortemente para essas variações.

A média geral dos cultivos protegidos $(105,49 \mathrm{t} / \mathrm{ha})$ foi superior à média dos cultivos a céu aberto $(91,84 \mathrm{t} / \mathrm{ha})$, o que era de se esperar. Resultados semelhantes foram constatados por Mills et al. (1990), Reis et al. (1991), Araújo et al. (1995), Fontes et al. (1997) e Lopes (1997). Entretanto, observou-se que em dois ambientes $\left(\mathrm{SC}_{5} \mathrm{e} \mathrm{SH}\right)$ ocorreram médias inferiores à média geral dos cultivos a céu aberto. No sistema hidropônico (SH), tal fato deveuse a falhas, principalmente no preparo da solução nutritiva, fato esse justificável, por ser uma técnica recente com a cultura do tomateiro no Brasil. Já no ambiente $\mathrm{SC}_{5}$, a alta incidência de nematóides na área deste ensaio, foi, provavelmente, o fator que mais contribuiu para os baixos rendimentos médios.

À exceção dos ambientes $\mathrm{SC}_{2}$ (cultivo protegido) e $\mathrm{CA}_{2}$ (céu aberto), nos demais não ocorreram diferenças significativas entre as cultivares, dentro de cada sistema de cultivo. As cultivares Donador, no ambiente $\mathrm{CA}_{2}$ e HE-295, no ambiente $\mathrm{SC}_{2}$, apresentaram produtividades inferiores às cultivares Vita $\mathrm{e}$ Carmen, respectivamente.

O peso médio de fruto é um relevante componente da produção, além de ser a melhor maneira de exprimir, indiretamente, o tamanho dos frutos. Houve ampla variação, em termos de cultivares e de ambientes (Tabela 3). Ao contrário do observado quanto à produtividade, o maior peso médio de frutos foi obtido nos cultivos a céu aberto $(159,80 \mathrm{~g})$, em relação aos cultivos protegidos $(142,40 \mathrm{~g})$.

Resultados semelhantes foram encontrados por Oliveira (1995) e Lopes (1997). A provável explicação é que nos cultivos protegidos, a densidade de plantas é maior, e, conseqüentemente, ocorre uma maior competição entre as plantas (Fery \& Janick, 1970). Nessa característica, só não ocorreram diferenças significativas entre cultivares dentro de cada sistema de cultivo nos ambientes $\mathrm{SC}_{1}$ e $\mathrm{SC}_{4}$.

A principal fonte de variação foi representada pelos ambientes, apesar de também ter sido obtida significância com relação a genótipos (Tabela 4). A interação genótipos $\mathrm{x}$ ambientes foi significativa quanto a ambas as características, evidenciando, assim, que as cultivares apresentaram comportamentos diferenciados diante da variação ambiental, o que indica a necessidade de se realizar um estudo para identificar os materiais de maior estabilidade.

Houve grande influência de ambientes dentro de genótipos, explicada pela regressão linear, pois o valor do efeito de ambiente linear foi 147 e 29 vezes maior que o da interação com ambientes, quanto à produtividade e ao peso médio dos frutos, respectivamente. Quanto à interação genótipos x ambientes (linear), somente para o peso médio esta fonte de variação mostrou-se significativa $(\mathrm{P}<0,01)$, e, segundo Santos (1980), a ocorrência da interação (linear) indica a existência de diferenças genéticas entre as cultivares, com relação aos seus comportamentos, o que pode ser constatado pela diferença entre os coeficientes de regressão das cultivares. Os desvios combinados também mostraram-se altamente significativos quanto ao peso médio dos frutos, o que sugere que os componentes linear e não-linear de estabilidade estão envolvidos no desempenho fenotípico das cultivares nos ambientes estudados.

Houve comportamento diferenciado em face das mudanças ambientais em relação a adaptabilidade e estabilidade das cultivares (Tabela 5).

Tabela 4. Resumo das análises de variância conjuntas pelo método de Eberhart \& Russell (1966), das seis cultivares de tomateiro nos nove ambientes, referentes às características selecionadas, destacando o quadrado médio e a significância pelo teste F. Unimar, Marília, 1997/1998.

\begin{tabular}{lrcc}
\hline Causa de variação & GL & \multicolumn{2}{c}{ Quadrado médio } \\
\cline { 2 - 4 } & & $\begin{array}{c}\text { Produção } \\
\text { comerciável }\end{array}$ & $\begin{array}{c}\text { Peso médio } \\
\text { de frutos }\end{array}$ \\
\hline Genótipos (G) & 5 & $336,03^{* *}$ & $851,48^{* *}$ \\
Ambientes (A) & 8 & $1.995,54^{* *}$ & $1.145,35^{* *}$ \\
Interação GxA & 40 & $85,07^{*}$ & $119,61^{* *}$ \\
Amb. dentro de genótipo & 48 & $403,48^{* *}$ & $290,57^{* *}$ \\
Ambientes (linear) & 1 & $15.964,34^{* *}$ & $9.162,76^{* *}$ \\
Interação GxA (linear) & 5 & $108,52^{\text {ns }}$ & $312,57^{* *}$ \\
Desvios de regressão & & & \\
Carmen & 7 & $64,89^{\text {ns }}$ & $49,11^{\text {ns }}$ \\
Diva & 7 & $18,18^{\text {ns }}$ & $37,98^{\text {ns }}$ \\
Donador & 7 & $92,37^{\text {ns }}$ & $147,54^{* *}$ \\
Graziela & 7 & $43,81^{\text {ns }}$ & $84,37^{* *}$ \\
HE-295 & 7 & $111,74^{*}$ & $123,99^{* *}$ \\
Vita & 7 & $77,63^{\text {ns }}$ & $17,25^{\text {ns }}$ \\
Desvios combinados & 42 & $68,10^{\text {ns }}$ & $76,71^{* *}$ \\
\hline Resíduo médio & 135 & 49,97 \\
\hline nsNão-significativo. ${ }^{*}$ e $*$ Significativo a $5 \%$ e a $1 \%$ de probabilidade, res- \\
pectivamente, pelo teste F.
\end{tabular}

Pesq. agropec. bras., Brasília, v. 37, n. 1, p. 81-88, jan. 2002 
Tabela 5. Estimativas dos parâmetros de adaptabilidade de $\left(\mu_{\mathrm{i}}, \mathrm{b}, \mathrm{S}_{\mathrm{di}}^{2}\right.$ e $\left.\mathrm{R}^{2}\right)$ para as características de seis cultivares de tomateiro, obtidas pelo método de Eberhart \& Russel (1966). Unimar, Marília. 1997/1998.

\begin{tabular}{|c|c|c|c|c|c|c|c|}
\hline $\begin{array}{l}\text { Parâmetros de } \\
\text { adaptabilidade } \\
\text { e estabilidade }\end{array}$ & Carmen & Diva & Donador & Graziela & HE-295 & Vita & Média \\
\hline & \multicolumn{7}{|c|}{ Produtividade de frutos comerciáveis (t/ha) } \\
\hline$\mu^{(1)}$ & $105,58 \mathrm{ab}$ & $94,01 \mathrm{~b}$ & $107,34 \mathrm{a}$ & $95,42 \mathrm{ab}$ & $96,93 \mathrm{ab}$ & $106,38 \mathrm{ab}$ & 100,94 \\
\hline$b_{i}$ & 1,000 & 0,815 & 1,304 & 0,741 & 1,063 & 1,081 & 1,00 \\
\hline$S_{d i}^{2}$ & 14,92 & $-31,79$ & 42,39 & $-6,17$ & $61,77^{*}$ & 27,66 & \\
\hline $\mathrm{R}^{2}$ & $0,85 * *$ & $0,93 * *$ & $0,87 * *$ & $0,83 * *$ & $0,79 * *$ & $0,85 * *$ & \\
\hline & \multicolumn{7}{|c|}{ Peso médio de frutos $(\mathrm{g})$} \\
\hline$\mu$ & $136,02 \mathrm{c}$ & $154,86 \mathrm{ab}$ & $141,68 b c$ & $142,52 \mathrm{bc}$ & $152,34 \mathrm{ab}$ & $161,92 \mathrm{a}$ & 148,22 \\
\hline$b_{i}$ & $0,369 * *$ & 1,039 & 0,788 & 0,861 & $1,711^{*}$ & 1,233 & 1,00 \\
\hline$S_{d i}^{2}$ & 24,21 & 13,07 & $122,63 * *$ & $59,47 * *$ & $99,08 * *$ & $-7,65$ & \\
\hline $\mathrm{R}^{2}$ & 0,38 & $0,86^{* *}$ & $0,48^{*}$ & $0,66^{* *}$ & $0,84 * *$ & $0,95 * *$ & \\
\hline
\end{tabular}
respectivamente.

Quanto à produtividade dos frutos comerciáveis, somente a cultivar Donador apresentou coeficiente de regressão diferente da unidade $\left(b_{i} \neq 1,0\right)$, o que a caracteriza com desempenho mais do que proporcional à melhoria promovida no ambiente, e, como apresentou produtividade acima da média geral e comportamento previsível, poderia ser indicada para ambientes considerados favoráveis. As demais cultivares apresentaram coeficientes de regressão igual à unidade, caracterizando-se como dotadas de desempenho proporcional à melhoria ambiental, e, quando associadas a alta produtividade, podem ser classificadas como de adaptabilidade geral nos ambientes estudados. As cultivares Carmen e Vita apresentaram ampla adaptabilidade a todos os ambientes estudados, uma vez que mostraram produtividades superiores à média geral, e quanto à estabilidade, apresentaram $\mathrm{S}_{\mathrm{di}}^{2}$ igual a zero, portanto, alta previsibilidade de comportamento, ou seja, são capazes de manter um comportamento semelhante ao apresentado, se cultivadas em ambientes parecidos aos do presente estudo.

As cultivares Diva, Graziela e HE-295 também apresentaram coeficientes de regressão que não diferiram da unidade, porém, como apresentaram baixas produtividades, podem ser classificadas como de pobre adaptabilidade nos ambientes estudados. Já a cultivar HE-295 foi a única a apresentar desvios da regressão significativo $\left(\mathrm{S}_{d i}^{2} \neq 0\right)$, possuindo baixa previsibilidade frente às oscilações ambientais, sendo considerada, portanto, a única não estável do grupo de cultivares avaliadas. Os valores dos coeficientes de determinação $\left(\mathrm{R}^{2}\right)$ mostraram, de maneira geral, um adequado ajustamento dos dados à reta de regressão.

Para a característica peso médio dos frutos, Carmen apresentou um dos menores valores entre os materiais estudados, coeficiente de regressão significativo, e muito abaixo de unidade $\left(b_{i}=0,369\right)$, revelando, assim, adaptação às condições desfavoráveis de cultivo. Além disso, deve-se ressaltar que não houve um bom ajustamento dos dados, ao modelo utilizado neste trabalho, em função do baixo valor do coeficiente de determinação $\left(\mathrm{R}^{2}=0,38\right)$. $\mathrm{O}$ genótipo HE-295 apresentou coeficiente de regressão significativo e acima da unidade, indicando que possui grande capacidade de responder vantajosamente aos estímulos ambientais.

As cultivares Donador, Graziela e HE-295 apresentaram desvios da regressão significativos, indicando, portanto, serem estes instáveis face às alterações ambientais. As cultivares Diva e Vita foram as únicas que mostraram ampla adaptação $\left(b_{i}=1,0\right)$ a todos os ambientes, comportamento altamente previsível ( $\mathrm{S}_{\mathrm{di}}^{2}=0$ ), além de apresentarem pesos médios de frutos superiores aos da média geral $\left(\mu_{\mathrm{i}}>148,22 \mathrm{~g}\right)$.

\section{Conclusões}

1. A análise da interação genótipos $x$ ambientes pelo método de Eberhart \& Russell é eficiente na discriminação do desempenho individualizado das cultivares, em face das variações ambientais. 
2. As cultivares Carmen, Donador e Vita apresentam rendimento médio superior ao da média geral dos ensaios, adaptabilidade geral e comportamento previsível em todos os ambientes estudados.

3. As cultivares Diva e Vita apresentam peso médio dos frutos superior aos da média geral, e são as únicas que possuem ampla adaptabilidade a todos os ambientes e comportamento altamente previsível.

\section{Referências}

ANNICCHIARICO, P. Cultivar adaptation and recommendation from alfalfa trials in Northern Italy. Journal of Genetics \& Breeding, Roma, v. 46, n. 1, p. 269-278, 1992.

ARAÚJO, M. L.; OLIVEIRA, A. C. B.; FERREIRA, J. M.; LEAL, N. R. Efeito da cobertura plástica sobre a qualidade de frutos de tomateiro na região norte fluminense. Horticultura Brasileira, Brasília, v. 13, n. 1, p. $68,1995$.

BERRY, S. Z.; UDDIN, M. R.; GOULD, W. A.; BISGES, A. D.; DYER, G. D. Stability in fruit yield, soluble solids and citric acid of eight machine-harvested processing tomato cultivars in Northern Ohio. Journal of the American Society for Horticultural Science, Alexandria, v. 113 , n. 4, p. 604-608, 1988.

CONTI, S.; SANGUINETI, M. C.; RONCARATI, R. Hybrid performance as compared to parents in processing tomato. Advances in Horticultural Science, Florence, v. 4, n. 3, p. 151-154, 1990.

CRUZ, C. D.; TORRES, R. A. de A.; VENCOVSKY, R. An alternative approach to stability analysis proposed by Silva and Barreto. Revista Brasileira de Genética, Ribeirão Preto, v. 12, n. 3, p. 567-580, 1989.

DELLA VECCHIA, P. T.; KOCH, P. S. Tomate longa vida: O que são, como foram desenvolvidos? Horticultura Brasileira, Brasília, v. 18, n. 1, p. 3-4, 2000.

EBERHART, S. A.; RUSSELL, W. A. Stability parameters for comparing varieties. Crop Science, Madison, v. 6, n. 1, p. 36-40, 1966.

FAO (Roma, Itália). Agricultural production: primary crops: tomato. Disponível em: <http://www. fao. org $>$. Acesso em: 29 jan. 2000.

FERY, R. L.; JANICK, J. Effect of planting pattern and population pressure on the yield response of tomato. HortScience, Alexandria, v. 5, n. 5, p. 443-444, 1970.
FINLAY, K. W.; WILKINSON, G. N. The analysis of adaptation in a plant breeding programme. Australian Journal of Agricultural Research, Melbourne, v. 14, n. 4, p. 742-754, 1963.

FONTES, P. C. R.; DIAS, E. N.; ZANIN, S. R.; FINGER, F. L. Produção de cultivares de tomate em casa de vegetação coberta com plástico. Revista Ceres, Viçosa, MG, v. 44, n. 252, p. 152-160, 1997.

GULL, D. D.; STOFFELlA, P. J.; OLSON, S. M.; LOCASCIO, S. J.; BRYAN, H. H.; EVERETT, P. H.; HOWE, T. K.; SCOTT, J. W. Quality of Florida fresh market tomato genotypes as affected by production environment. Proceedings of the Florida State Horticultural Society, Gainesville, v. 97, p. 140-142, 1984.

LIN, C. S.; BINNS, M. R. A method of analyzing cultivar x location $\mathrm{x}$ year experiments: a new stability parameter. Theoretical and Applied Genetics, Berlin, v. 76, n. 3, p. 425-430, 1988.

LOPES, P. R. A. Influência da cobertura do solo e sistema de condução das plantas, na cultura do tomateiro (Lycopersicon esculentum Mill.) cultivado em casa-devegetação e no campo. Jaboticabal: Unesp, 1997. 125 p. Tese de Doutorado.

MAKISHIMA, N.; MIRANDA, J. E. C. Cultivo do tomate. Brasília: Embrapa-CNPH, 1995. 22 p. (Instruções Técnicas, 11).

MILLS, P. J. W.; SMITH, I. E.; MARAIS, G. A greenhouse design for a cool subtropical climate with mild winters based on microclimatic measurements of protected environments. Acta Horticulturae, Leuven, n. 281, p. 83-94, 1990.

MORAES, C. A. G. Hidroponia: como cultivar tomates em sistema NFT (técnica de fluxo laminar de nutrientes). Jundiaí: DISK, 1997. 141 p.

OLIVEIRA, M. R. V. O emprego de casas-de-vegetação no Brasil: vantagens e desvantagens. Pesquisa Agropecuária Brasileira, Brasília, v. 30, n. 8, p. 10491060, ago.1995.

ORTIZ, R. Una metodología de selección múltiple por productividad y estabilidad para cultivares de tomate. Agrociencia, Ibadan, v. 7, n. 2, p. 135-142, 1991.

ORTIZ, R.; IZQUIERDO J. Yield stability differences among tomato genotypes grown in Latin America and the Caribbean. HortScience, Alexandria, v. 29, n. 10, p. 1175 1177, 1994.

PEIXOTO, N.; MENDONÇA, J. L.; SILVA, J. B. C. da; BARBEDO, A S. C. Rendimento de cultivares de tomate 
para processamento industrial em Goiás. Horticultura Brasileira, Brasília, v. 17, n. 1, p. 54-57, mar. 1999.

POYSA, V. W.; GARTON, R.; COURTNEY, W. H.; METCALF, J. G.; MUEHMER, J. Genotypeenvironment interactions in processing tomatoes in Ontario. Journal of the American Society for Horticultural Science, Alexandria, v. 111, n. 2, p. 293-297, 1986.

REIS, N. V. B.; HORINO, Y.; OLIVEIRA, C. A. S.; BOITEUX, L. S. Influência dos parâmetros agrometeorológicos sobre a produção de nove genótipos de tomate plantados a céu aberto e sob proteção de casade-vegetação plástica. Horticultura Brasileira, Brasília, v. 9 , n. 1 , p. $55,1991$.
SANTOS, J. B. dos. Estabilidade fenotípica de cultivares de feijão (Phaseolus vulgaris L.) nas condições do Sul de Minas Gerais. Piracicaba: Esalq, 1980. 109 p. Dissertação de Mestrado.

VOICAN, V.; LACATUS, V.; TANASESCU, M.; FERNANDEZ MUÑOZ, R.; CUARTERO, J.; GOMEZ GUILLAMON, M. L. Growth and development of tomato plants related to climatic conditions from some areas of Romania. Acta Horticulturae, Leuven, n. 412, p. $355-365,1995$.

WILLIAMS, W.; GILBERT, N. Heterosis and the inheritance of yield in tomato. Heredity, Oxford, v. 14, n. 1 , p. $133-149,1960$. 